\title{
Brazil nut effect and excluded volume attraction in vibrofluidized granular mixtures
}

\author{
M. Bose, U. U. Kumar, P. R. Nott, and V. Kumaran \\ Department of Chemical Engineering, Indian Institute of Science, Bangalore, India
}

\begin{abstract}
A two dimensional bi-disperse vibrofluidized granular mixture is studied in the rapid flow regime, where particle interactions occur due to instantaneous collisions. Both experiments and simulations are carried out, and these show the existence of two phenomena which have been observed only in very dense granular flows or in equilibrium systems. The Brazil nut phenomenon, which involves the rise of larger particles in a granular mixture upon vibration, has been observed in dense systems due to the percolation of small particles though the interstitial spaces between the large particles, or due to convection rolls. In the present case, where neither effect is present, it is observed that the fluidization of the smaller particles by vibration results in an exponentially decaying density profile, at heights large compared to the particle diameter, and thereby a pressure field that decreases with height. The larger particles, suspended in this decaying pressure field, experience a larger pressure at the bottom and a smaller pressure on top, and they rise to a height where the net force caused by the decreasing pressure is balanced by the weight of the particle. An attractive force between the large particles, similar to the entropic attraction effect in mixtures of colloids and polymers, is also observed in this nonequilibrium system, because when the distance between the large particles is less than the small particle diameter, the pressure between the large particles is smaller than that on the outside. Analytical results are derived for each of these effects, and these are in agreement with the experimental and simulation results.
\end{abstract}

\section{INTRODUCTION}

The present study deals with a binary mixture of inelastic particles under vertical vibration. An interesting phenomenon observed in a vibrated binary mixture is the segregation of the mixture. It is observed that larger particles "rise" to the top, and the smaller particles settle to the bottom, and this phenomenon is known as the "Brazil nut phenomenon (BNP)" in the literature. However, the reverse phenomena is also observed and is named as "reverse buoyancy" or "reverse Brazil nut phenomenon (RBNP)." Several reasons have been identified for BNP, including the percolation of particles [1], the reorganization of particles [2-4], and the presence of large convection rolls in the system $[5,6]$. Most of these studies considered a single large particle in a background of small particles, where the density of the small particles are high and the "temperature" (which is the mean square velocity of the small particles) is low compared to the product of the gravitational acceleration and particle diameter. A recent experiment [7] reported reverse buoyancy in a vibrated granular mixture where they observed the heavier of the larger particles rise in the sea of the smaller particles but equally large light particles sink. Numerical simulations have [8] reported a "condensation" driven segregation, and proposed a theory to determine the crossover from the BNP to RBNP based on the competition between the percolation and condensation of hard spheres. This phenomenon has been experimentally realized by Ref. [9] (see references therein for the controversy on experimental realization of the phenomenon).

Recent experiments revealed another interesting phenomenon in vibrated granular mixtures. It has been observed $[10,11]$ that equipartition of energy does not apply in a vibrated granular mixture of same size but different mass. They have also observed that the mass difference has a stron- ger effect on nonequipartition of energy than the difference in the coefficients of restitution of particles. Apart from the experiments and simulation focused mainly on the segregation, there are a number of studies where the details of different microscopic and macroscopic properties of a binary mixture is studied for both vibrated and shear flow $[12-14,18]$. The reader is referred to Refs. $[12,14]$ and references therein for further details.

There are two areas of focus in the present study. The first is the "rise" of larger particles observed in a background of smaller particles when the smaller particles are in a fluidized state. The reason for this rise is very different from the percolation and circulation effects, and is due to the equilibration of the larger particle in a decreasing pressure gradient created by the smaller particles as reported in Sisodia and Wassgren [19]. We report the rise of the larger particle in a back-ground of smaller particles in an experimental vibrofluidized bed, and compare the experimental results with the results of a simple model.

Segregation in granular mixtures, which occurs when an initially homogeneous mixture separates into its constituents, is of great industrial interest, since solids handling and transport constitutes a significant fraction of industrial operations. While segregation has been widely observed in experiments $[7,15]$, the mechanisms leading to segregation are still poorly understood. Segregation in binary mixtures has been observed [16] in horizontally shaken granular layers, and it has been observed that the onset of segregation has the features of a continuous phase transition. It has been speculated [16] that one of the reasons for segregation could be the attractive force due to the excluded volume interaction between large particles in a background of agitated small particles. Aumaitre et al. [17] observed clustering in a horizontally vibrated monolayer of particles consisting of large discs and small spheres. They showed that there is an asymmetry in the pres- 
sure experienced by the larger particles due to the excluded volume effect, and also measured increases in the segregation velocity with the frequency of vibration. However, a quantitative characterization of the excluded volume effect was complicated by the frictional force between the particles and the base. The second focus of the present study is the attractive force between particles which leads to segregation. This attractive force is determined from the pair distribution function in a two dimensional vibrofluidized bed, and this provides conclusive evidence that there does exist an attractive force at the microscopic scale. The magnitude of the attractive force is determined from the pair distribution function for the particles, and not on the basis of the macroscopic segregation phenomenon. A model, similar to that for the entropic aggregation in colloidal suspensions, is developed for this phenomenon.

In the case of the excluded volume attraction, we examine the analogy between a thermal equilibrium system, which is a pair of large particles in a background of small particles fluctuating due to thermal energy, and a driven system, which consists of a pair of large particles in a background of small particles fluctuating due to external driving by a vibrator. There are two aspects to this analogy. The first is the calculation of the potential due to the excluded volume interaction. The potential $U$ due to excluded volume effects can be calculated using mechanical force balance arguments [20] as described above. The second aspect is the increase in the pair distribution function due to the potential. In a dilute system of particles with pair potential $U$, thermodynamic arguments can be used to show that the pair distribution function at contact is $\exp (-U / T)$, where $T$ is the thermal energy (product of the Boltzmann constant and temperature). If the system consists of hard spheres which have a weak attractive potential, the pair distribution function at contact is that for hard spheres multiplied by a factor $\exp (-U / T)$. In the present experiments and simulations, we examine whether a similar relation applies for the driven system, where the fluctuating energy of the small particles is due to external driving, instead of thermal fluctuations.

\section{EXPERIMENTAL AND SIMULATION METHODS}

A mixture of particles of two different sizes, spheres of 5 $\mathrm{mm}$ diameter, and disks of $10 \mathrm{~mm}$ diameter, are confined between two glass walls separated by a distance of $6 \mathrm{~mm}$ and thickness $200 \mathrm{~mm}$, and these are fluidized by a vibrating base (Fig. 1). The larger disks are designed so that the masses of the spheres and disks are comparable, and the friction between the glass wall and the disks is negligible. This is achieved by making the thickness larger near the outer edge and smaller at the center, as shown in Fig. 1(b). This reduces the mass of the disk, and the area of contact between the disk and glass walls. In addition, the thickness of the disk at the rim is slightly smaller than the distance between the glass plates, so that the disks are confined to move in the direction perpendicular to the glass plates. The particles are set into motion by a steel base plate of $5 \mathrm{~mm}$ thickness and $200 \mathrm{~mm}$ length, attached to a vibrator (Syscon instruments). The vi-

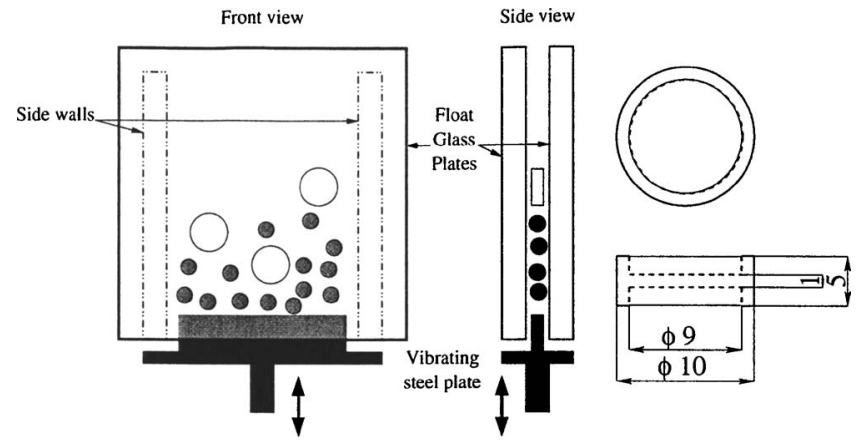

(a)

(b)

FIG. 1. Schematic of the setup (a) and the shape of the discs (b). In order to reduce the mass of the disks, a central annular portion of the particle is turned out. All dimensions are given in $\mathrm{mm}$.

brator can operate between $1 \mathrm{~Hz}$ and $1 \mathrm{kHz}$ and the maximum amplitude is $12 \mathrm{~mm}$, but the amplitude decreases as the frequency increases. The motion of the particles is filmed using a high speed camera (Weinberger SpeedCam Pro-lt) which can film at a maximum speed of 1000 frames per second with a spatial resolution of $512 \times 512$ pixels. The normal particle-wall coefficient of restitution, obtained by dropping a ball on the horizontal surface and measuring the pre- and postcollisional velocities, was found to be $0.9 \pm 0.02$ (95\% confidence level). This coefficient of restitution was used for particle-wall and particle-particle collisions in the simulations.

The simulation of bi-disperse particles basically follows the hard sphere event driven (ED) simulation technique discussed in standard references [21,22]. The ED simulation scheme consists of a streaming and a collision step. In the streaming step the time required for the next collision is calculated based on the current particle positions and velocities, and the positions are advanced along ballistic trajectories up to the next collision. In the collision step, the velocity of the colliding particle is updated following the collision rule.

The number of larger disks is fixed at 5 , so that there is very little direct interaction between the larger disks, and the interaction is mediated by the smaller spheres. The number of smaller spheres is varied in the experiments, and the amplitude and frequency of vibration is maintained at $0.85 \mathrm{~mm}$ and $30 \mathrm{~Hz}$, respectively. The density, mean square velocity, velocity distribution function, and the pair distribution function are determined using image analysis for each species. The results obtained from the experiment are verified with the results obtained from the simulations.

The amplitude and frequency of vibration were maintained at $0.85 \mathrm{~mm}$ and $30 \mathrm{~Hz}$ in the experiments. The experiments were carried out for a fixed diameter ratio $(0.5)$ and mass ratio, $(0.65)$ of the spheres and disks, both of which are made of steel. The size and mass ratios could not be varied easily in the experiments due to limitations in fabrication. However, the experimental results were verified with two dimensional event driven simulations, and results were also obtained from simulations for the cases where the mass ratio is 1 and the diameter ratio is small, as well as for cases where the diameter ratio is 1 and the mass ratio is small. The num- 
TABLE I. Parameters involved in the experiment. $N_{\mathrm{s}}$ : total number of small particles; $N_{\mathrm{b}}$ : total number of the large particles; $\phi$ : ratio of the small particles to the total number of particles; $N_{\text {ws }}$ : number of particles per unit width $\left(\mathrm{m}^{-1}\right)$.

\begin{tabular}{cccc}
\hline \hline$N_{\mathrm{s}}$ & $N_{\mathrm{b}}$ & $\begin{array}{c}\phi \\
N_{\mathrm{s}} / N_{\mathrm{t}}\end{array}$ & $\begin{array}{c}N_{\text {ws }} \\
m^{-1}\end{array}$ \\
\hline 25 & 5 & 0.833 & 125 \\
50 & 5 & 0.9090 & 250 \\
75 & 5 & 0.9375 & 375 \\
100 & 5 & 0.952 & 500 \\
125 & 5 & 0.9615 & 625 \\
150 & 5 & 0.9677 & 750 \\
\hline \hline
\end{tabular}

ber of larger disks was fixed at 5 in all the experiments, to ensure that there is very little direct interaction between the disks, while the number of smaller particles were varied in order to vary the density and temperature profiles of the smaller particles. The numbers of large and small particles used in the experiment are shown in Table I. In order to validate the technique used for obtaining the density profiles, the local number density (number per unit area), obtained from the area fraction profiles, were integrated over the height of the bed to calculate the number of particles per unit width. This was then compared with the number of particles inserted into the bed and the difference between the two was less than $0.1 \%$ of the number of particles.

\section{RESULTS AND DISCUSSION}

Figure 2 shows the density profiles of both the components in the mixture. The smaller particles show an initial increase and then an exponential decrease in density with height from the bottom vibrating wall. The density profiles for the larger particles also show the similar nature when the number of the smaller particles is small. However, the profile

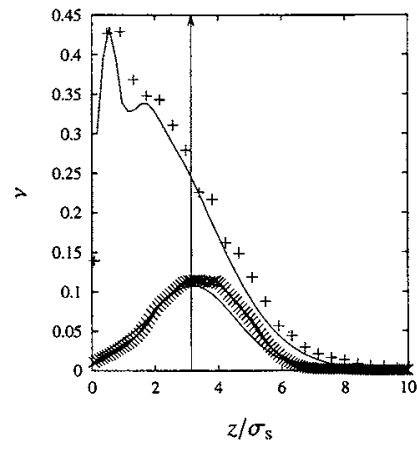

(a)

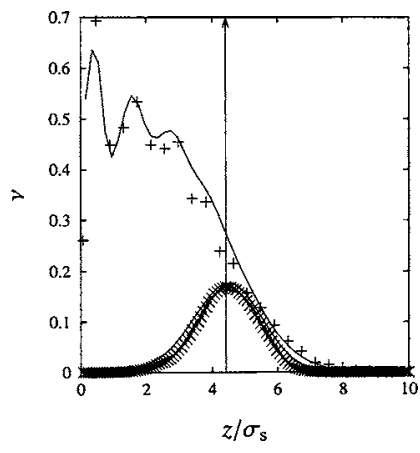

(b)
FIG. 2. Density profiles for both types of particles, obtained from simulation and experiment. The number of big particles is 5 and the number of small particles is varied, (a) $N_{\text {ws }}=375$, (b) $N_{\text {ws }}=625$. The vertical line marks the mean position of the big particles. $(+)$ and $(\times)$ represent the experimental data for small and big particles, respectively, and (-) represents the corresponding simulation results.

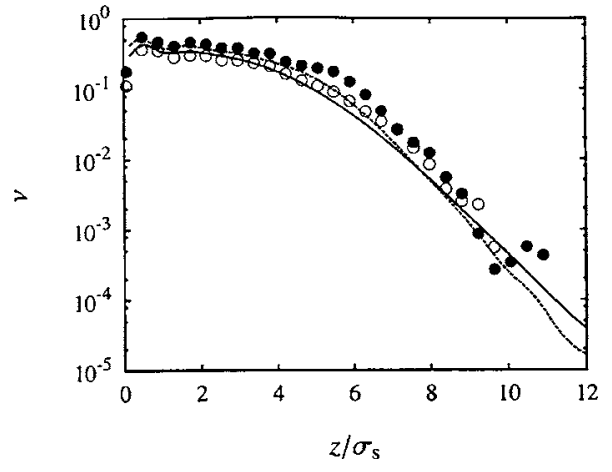

FIG. 3. Density profiles obtained from simulation (lines) and experiments (points) for different number density of the smaller species for mass ratio 0.65 . ( $\bigcirc)$ and $(-) N_{\mathrm{ws}}=375,(\bigcirc)$ and $(--)$ $N_{\text {ws }}=625$.

changes significantly as the number of smaller particle increases. Figure 2 compares the density profiles of the individual components obtained from experiment and simulation. A good agreement between the results of the simulation and the experiments is observed with no adjustable parameters. Density profiles for the smaller particles obtained from the simulations are plotted in a log-linear scale in Fig. 3. The experimental data could not be obtained at greater heights due to the lack of samples for statistical sampling. However, the simulation data clearly shows that the profile tends to a vertical line on a log-linear scale as height is increased, indicating that the density decreases exponentially with height.

We now compare the temperatures in the vertical and horizontal directions, which are the products of the masses and the mean square velocities, of the components in the mixture obtained from experiments and simulation. The velocity distribution and different moments of the velocity distribution are obtained by averaging over $10^{4}$ frames. Figure 4 shows the temperature for the small particles, scaled by the small particle mass, which is equivalent to the mean square velocities of the small particles, obtained from the simulation and the experiment for two different cases. The results obtained from experiments are in good agreement with the results obtained from the simulation. The temperatures of the small and large particles obtained from simulations were also compared with each other. It was not possible to obtain the temperature of the larger particles as a function of height from experiments, because the number of large particles was too small to obtain reliable averages. However, the temperatures could be obtained from simulations, as shown in Fig. 4. The simulation results show that both species have temperatures close to each other, because the coefficient of restitution is close to 1 and the particles are nearly elastic.

In Fig. 2, the density profile for the larger and smaller particles are shown as a function of height scaled by $\sigma_{\mathrm{s}}$, the diameter of the small particle. In this case, it is seen that the mean height of the larger particles, indicated by the straight line, is larger than the mean height of the smaller particles, indicating that the flow results in an upward segregation of the larger particles. This is seen more clearly in Fig. 5, where the density profile of bigger particles is shown for different number densities of smaller particles. It is observed that 


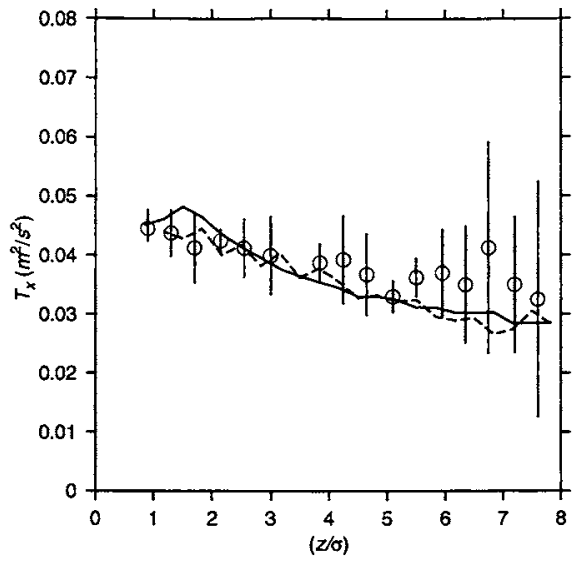

(a)
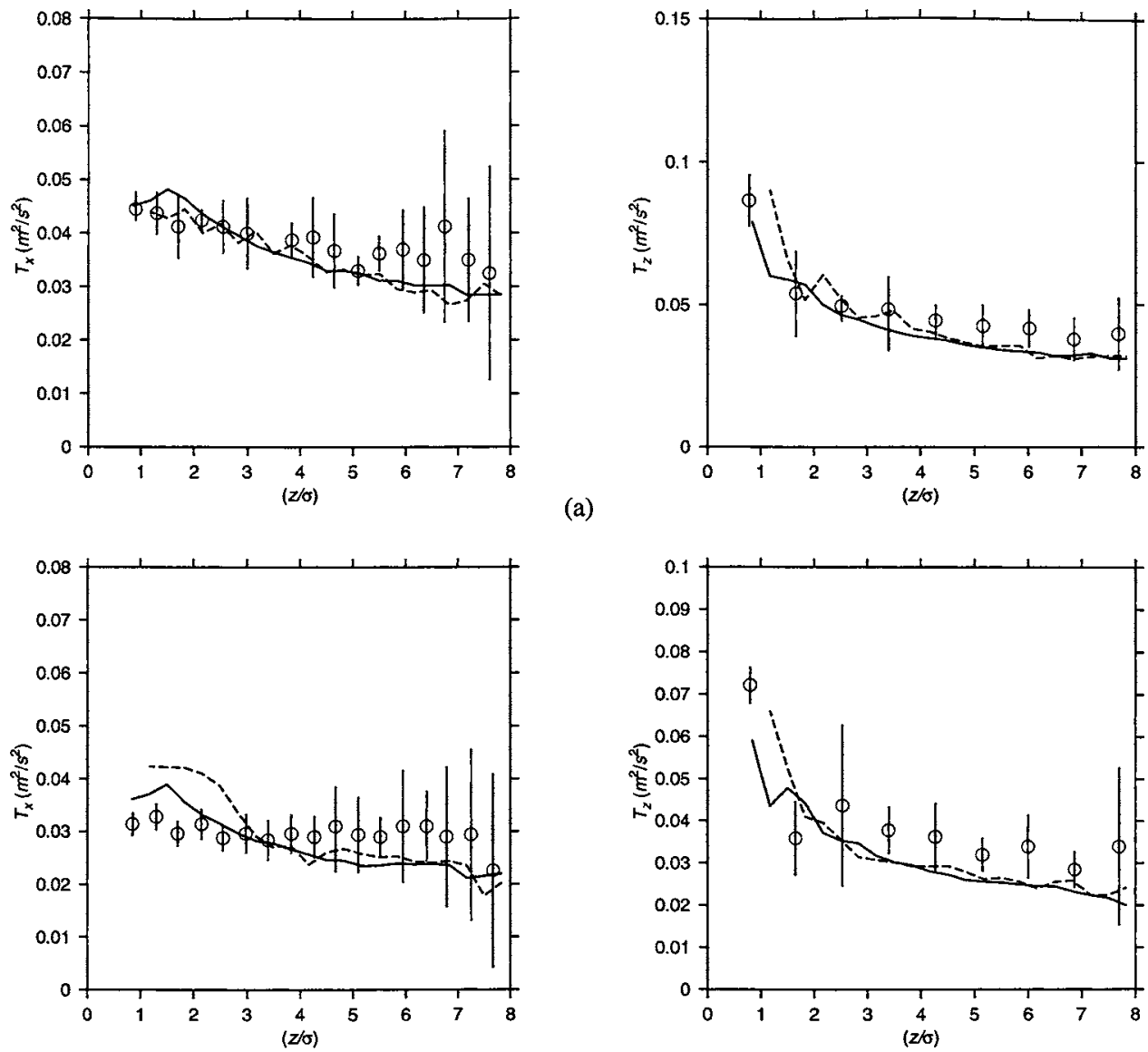

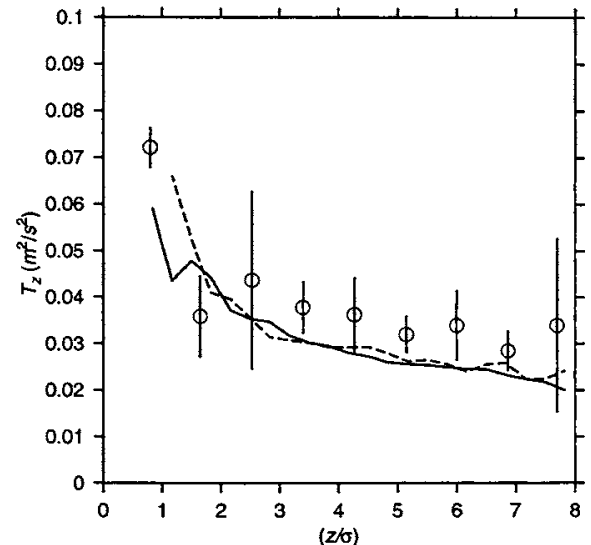

FIG. 4. Temperature profiles, scaled by the mass of the smaller particles, for two different number densities, (a) $N_{\mathrm{s}}=100, N_{\mathrm{ws}}=500$, and $\phi=0.9375$, and (b) $N_{\mathrm{s}}=125$, $N_{\text {ws }}=625$, and $\phi=0.9615$. ( $\left.\bigcirc\right)$ Experimental results, and (-) simulation results for small particles, $(-\quad-)$ simulation results for large particles.

(b)

when the density of the smaller particles is small, the density profile of the larger and smaller particles are qualitatively similar. However, when the density of the smaller particles increases, the mean position of the larger particles shifts upward, resulting in a segregation.

That larger particles have a tendency to "rise" in a vibrated granular mixture is a well studied problem and is

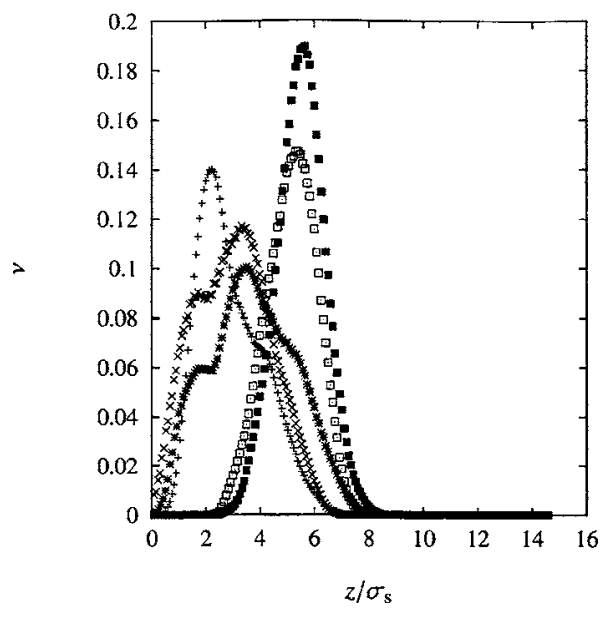

FIG. 5. Density profile of big particles for different number density of small particles, the number of the big particles is kept constant. (+) $N_{\mathrm{S}}=50,(\times) N_{\mathrm{s}}=75,(*) N_{\mathrm{s}}=100,(\square) N_{\mathrm{S}}=125$, $N_{\mathrm{s}}=150$. known in the literature as the "Brazil nut" phenomenon. Earlier studies [1-6] deal with the system with low temperature and/or with the systems where the small particles are in a convective motion. The cases that are presented here fall in neither of the above categories, but is due to the equilibration of the larger particle in the decreasing pressure gradient of the smaller particles, as reported by Sisodia and Wassgren [19].

The rise of the larger particle can be explained using a force balance on the large disk. It should first be noted that the density of the smaller particles first increases and then decreases with height, while the temperature first decreases and then attains a constant value. Therefore the pressure, which is the product of the density and temperature in the limit of low density, decreases with height. If a large particle is placed in the background of decreasing pressure, the pressure on the lower surface is larger, on average, than the pressure on the upper surface, and there is a net upward force due to the effect of the small particles. The equilibrium position of the larger particle is the position at which this upward force balances the weight of the particle,

$$
F_{A, \text { up }}=\int d S p \mathbf{n} \cdot \mathbf{e}_{z}-\frac{m_{b}}{m_{s}} g .
$$

In the above relation, all the quantities are nondimensionalized with the mass of the small particles, and this explains the mass ratio $\left(m_{b} / m_{s}\right)$ in the second term on the right side. 


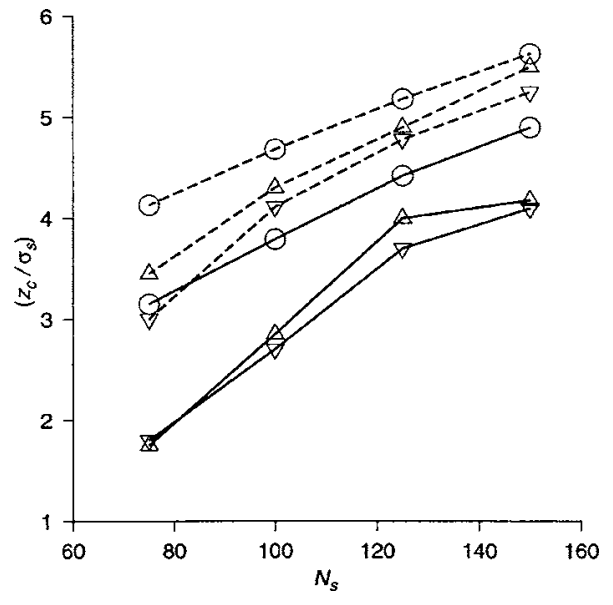

FIG. 6. Average height of the large particles scaled by the diameter of the small particles $\left(z_{c} / \sigma_{s}\right)$ as a function of the number of small particles. The solid lines show the results when the ratio of masses of the small and large particles is 0.65 , and the broken lines show the results when the ratio of masses is 1.0. $\bigcirc$ : results of experiments and simulations; $\triangle$ : results obtained using Eq. (1) in which the density profile is assumed to be exponential, and the temperature is calculated from the slope of the density profile (obtained from simulation) in a log-linear plot in the limit of large height; $\nabla$ : results obtained from Eq. (1) using the actual density and temperature profiles from simulations.

It should be noted that there is an average force only when the number density of the smaller particles is large, so that the larger particle experiences many collisions within a time period comparable to the relaxation time of the particle. Consequently, this effect is not observed when the density of the smaller particles decreases, and the frequency of collisions decreases.

The net upward force on a particle was calculated by two different methods. The first is to assume that the density profile is given by an exponential function (shown in Fig. 3), and use this to determine the pressure at the surface of the large particle and the net force. The temperature in the exponentially decaying region was determined by calculating the slope of the density profile on a log-linear plot, of the type shown in Fig. 4, and equating this to $(-g / T)$. The second is to use the numerical results of density and temperature profiles from simulations for calculating the pressure profile, and the net force. The results of the calculation were also compared with the results from experiments and simulations. The results of experiments and simulations are not provided separately, because the results are in agreement, as shown in Fig. 2. The mean position of the larger particles are shown in Fig. 6 for different numbers of the smaller particles. It is observed that the prediction of the mean position from a force balance and the mean position obtained from the simulations are not in a very good agreement, and there is a difference of about 10-30\% between the values predicted by the model and that obtained from simulation except for the first case. The result for $N_{s}=50$ is not provided in Fig. 6, because the mean position of the large particles is very close to the bottom, as shown in Fig. 5, and the pressure profile due to the smaller particle does not decrease in this region.

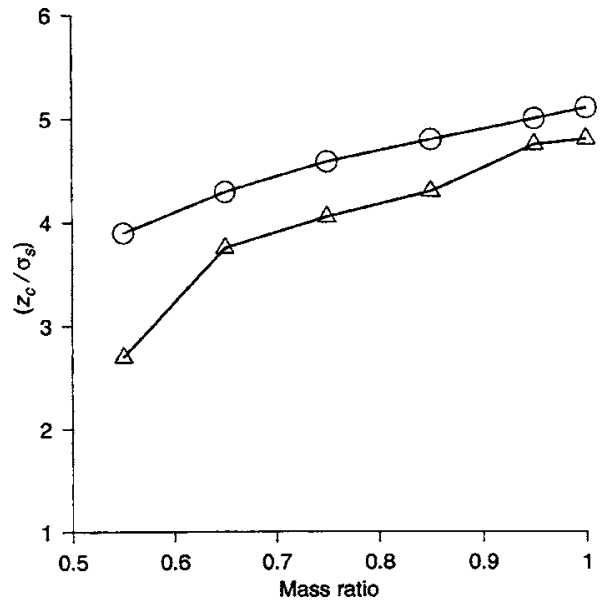

FIG. 7. Average height of the large particles scaled by the diameter of the small particles $\left(z_{c} / \sigma_{z}\right)$ as a function of the mass ratio of the particles when the number of smaller particles is 125 . $\bigcirc$ : results of experiments and simulations; $\triangle$ : results obtained from Eq. (1) using the actual density and temperature profiles from simulations.

It is to be noted here that the ratio of the mass of the smaller particles to that of the larger particles in the experiments is 0.65 , and this could not be easily varied due to limitations in fabrication. Some of the disagreement in Fig. 6 could be because the larger particle, being heavier, has an average position near the bottom of the bed where the density of the smaller particle is a nonmonotonic function of height, thereby making equilibration difficult. It was difficult to carry out experiments with particles of equal mass, due to limitations in fabrication. However, simulations were carried out for particles with equal mass and different sizes, and these indicate that the mean position of the large particles is higher than that in the previous case. This is because the masses of the larger and smaller particles are equal, and the buoyancy force is larger than for the case where the smaller particles are lighter than the larger ones. The force balance calculations were repeated, and the results from the are shown in Fig. 6. It is observed that the agreement between model predictions and simulations improves when the mass ratio increases, and the height of the center of mass increases. This is also shown in Fig. 7, where the variation of the position of the center of mass is shown as a function of the mass ratio when the number of small particles per unit width is 125 . It is observed that the agreement between model predictions and simulations improves as the mass ratio is increased, and the height of the center of mass increases.

From these studies, it is evident that the net upward motion of a larger particle in a mixture at high "temperature" is caused by the pressure gradient of the smaller particles. However, there is good agreement between model predictions and simulation only when the mass ratio of the larger and smaller particles is relatively high, so that the large particle equilibrates at a height where there is a decreasing density (and pressure) profile for the smaller particles. The agreement also becomes poor as the number of smaller particles is decreased, because the pressure decreases as the 


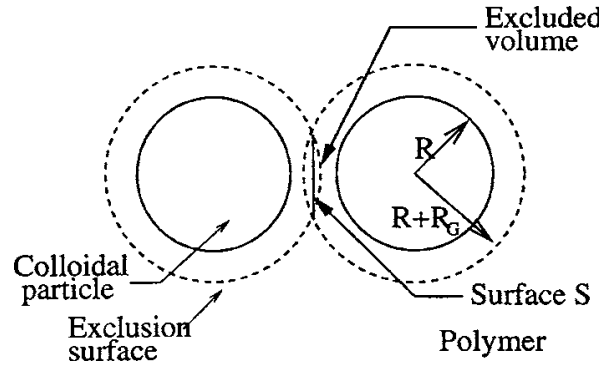

FIG. 8. Schematic of the excluded volume attraction in colloidal particles, showing the overlap between exclusion surface with radius $R+R_{G}$ around a colloidal particle of radius $R$ and the cross sectional area $S$ of the excluded volume. The attractive force between the particles is the osmotic pressure due to the polymer times the surface area $S$.

number density increases, and the pressure is not sufficient to balance the weight of the larger particles.

The area fraction profiles have multiple peaks, and the area fraction is close to 0.6 at the bottom of the bed. However, at the location of the peak of the density profile of the large particle, the area fraction of the small particle is small, so the rise of the large particle to this height cannot be due to the usual Brazil-nut mechanism. Even when the number of small particles is the lowest (375), the area fraction of the small particles at the location of the maximum of the large particle density profile is only 0.25 , and this further decreases as the number of small particles per unit area is increased. Though the system is dense at the bottom, there are no extended contacts and the system is definitely in the rapid flow regime at the location of the maximum of the density profile of the large particle.

\section{PAIR DISTRIBUTION FUNCTION}

The excluded volume attraction between colloidal particles in a polymer solution has been well studied, and has been explained on the basis of the exclusion of the polymer molecule from the region between the particles when the distance between the particles is less than the radius of gyration of the molecule. This force is usually referred to as the "entropic attraction" force due to the increase in the volume available to the polymers when the distance between the colloidal particles is less than the radius of gyration of the polymers. However, the original explanation put forth by Asakura and Osawa [20] for the excluded volume attraction force was a dynamical argument which made no reference to thermodynamic principles, and it is useful to revisit this argument and show the analogy with the present system. Consider a colloidal particle of radius $R$ in a dilute polymer solution, in which the polymer molecules have radius $R_{G}$, as shown in Fig. 8. The colloidal particles have a spherical "exclusion surface" of radius $\left(R+R_{G}\right)$ for the polymer molecules, and the centers of the nonadsorbing polymer molecules cannot enter this surface. If the distances between the surfaces of the particles is less than the radius of gyration of the polymer, so that the exclusion surfaces overlap, the polymer is excluded from the region between the particles. In this case, there is no osmotic pressure due to the polymer on the surfaces from which the polymer is excluded. However, there is an osmotic pressure on the outer surfaces where the polymers surround the particles. Therefore there is a force of attraction which is just proportional to the osmotic pressure times the cross sectional area $S$ of the region from which the polymers are excluded. The potential is then calculated by integrating this force with respect to distance.

A similar argument can be used for the vibrated granular material, except that the pressure in this case is not the osmotic pressure due to the polymer, but the pressure due to the fluctuating velocity of the smaller particles. If the distance between the surfaces of the larger particles is smaller than the diameter of the smaller particle, the smaller particles are excluded from the region between the larger particles, and there is no pressure due to the smaller particles in the region between the larger particles. There is a pressure, however, on the outer surfaces due to the smaller particles, and this causes an attractive force. If the pressure due to the smaller particles were a constant, the force would be just the product of the pressure and the area of cross section of the region from which the smaller particles are excluded. Since the pressure is not a constant, a slightly more complicated calculation is required to extract the force. The attractive potential is then obtained by integrating the force with respect to distance. Finally, it should be noted that the present configuration is two dimensional, so that the equivalent of the surface area $S$ for colloidal particles is just a line segment (denoted by $h$ in Fig. 11) in two dimensions.

In this section, we analyze the pair distribution function for the large particles in a background of small particles. For a homogeneous system, the pair distribution function $g(\mathbf{r})$ is defined such that $\rho^{2} g\left(\mathbf{r}_{1}, \mathbf{r}_{2}\right) d \mathbf{r}_{1} d \mathbf{r}_{2}$ is the probability of finding a pair of particles in the differential volumes $d \mathbf{r}_{1}$ and $d \mathbf{r}_{2}$ about the locations $\mathbf{r}_{1}$ and $\mathbf{r}_{2}$, respectively. Here, $\rho$ is the number density (number of particles per unit volume). For a system at equilibrium in which the interaction potential is pairwise additive, all thermodynamic properties can be expressed in terms of the pair potential [23]. The pair distribution function is only a function of the distance between the centers of the particles $r=\left|\mathbf{r}_{1}-\mathbf{r}_{2}\right|$ for spherically symmetric particles and for an isotropic medium, and is referred to as the "radial distribution function."

In the point particle approximation, where the volume fraction of the particles is very small, the radial distribution function is equal to 1 and is independent of the distance between the particles if the particles do not have any attractive interactions. At higher volume fraction, for hard disks and spheres in the absence of attractive interactions, the radial distribution function at contact (when the distance between the particle centers is equal to the particle diameter) increases, and is well approximated by the Verlet-Levesque [24] pair distribution function,

$$
g_{V L}(r=\sigma)=\frac{1-7 \nu / 16}{(1-\nu)^{2}}
$$

where $\nu$ is the area fraction in two dimensions. In the presence of attractive interactions, however, the pair distribution 


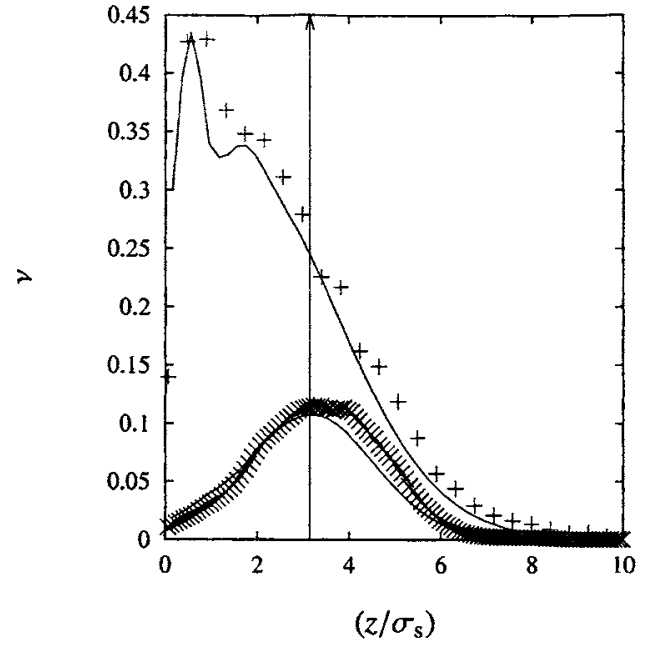

(a)

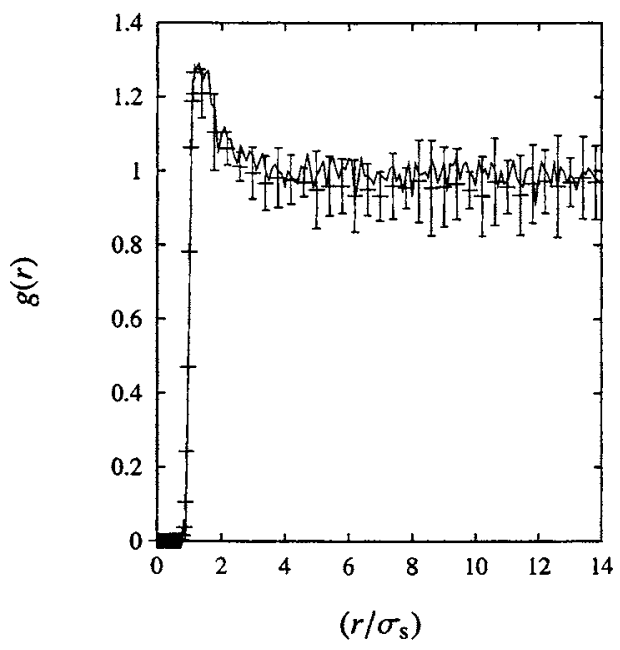

(c)

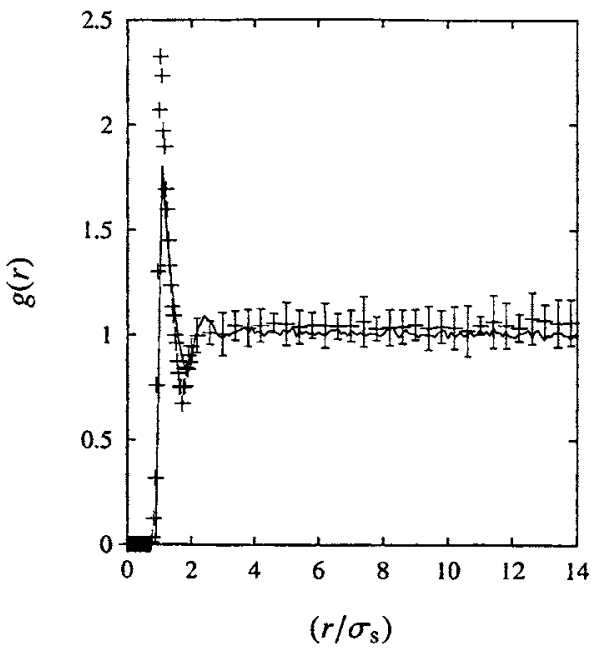

(b)

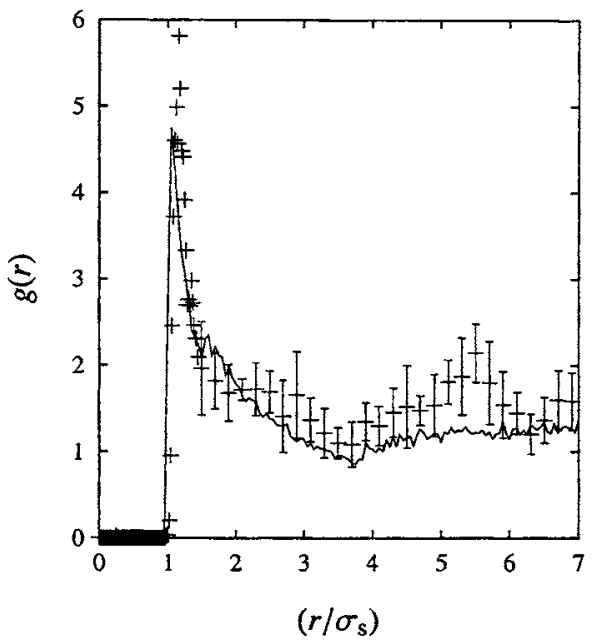

(d)
FIG. 9. $g(r)$ for $N_{w}=75$ for the small and big particles. (a) shows the density profiles for the small and large particles, (b) and (c) plot the $g(r)$ for small particles at $z / \sigma_{\mathrm{s}}=1$ and $z / \sigma_{\mathrm{s}}=3$, respectively, and (d) shows the $g(r)$ for the big particles at the vertical position where the concentration of large particles is a maximum. function at contact increases. For an equilibrium system, the radial distribution function is related to the pair potential energy in the dilute limit by [23]

$$
g(r)=\exp [-U(r) / T]
$$

where $T$ is the thermal energy (product of the Boltzmann constant and temperature) of the particles. In the presence of attractive interactions, the pair distribution function is given by

$$
g(r=\sigma)=g_{V L}(\nu) e^{-U(\sigma) / T}
$$

where, $g_{V L}(\nu)$ is the pair distribution function at contact given in Ref. [24] for the big particles in the absence of small particles. Thus the pair potential $U(\sigma)$ at contact between particles in the dilute limit can be inferred from the experimentally measured radial distribution function at contact using Eq. (3). For the dynamical system under consideration, we examine whether an equation of the type 3 applies, where $T$ is the mean square velocity of the particles, and whether the measured pair potential $U$ can be determined from simple kinetic theory arguments. (Note that the mass of the small particles is considered to be 1 without loss of generality, so that the mass dimension in all variables is scaled by the mass of the small particles, and the scaled potential has units of the square of the velocity.) Since there are two components in the present system, the pair distribution functions for the large and small particles, $g_{\mathrm{bb}}(r)$ and $g_{\mathrm{ss}}(r)$, are defined such that $g_{\mathrm{bb}}(r)$ is the probability density of finding a pair of big particles at a separation distance $r$, and $g_{\mathrm{ss}}(r)$ is the probability density of finding a pair of small particles separated by a distance $r$. As explained above, the focus of the analysis is on the pair distribution function of the larger particles, but we also examine the pair distribution function of the small particles to ensure that this is not significantly affected by the large particles.

The pair distribution function for the particles were calculated from the experiments as follows. The bed is divided into smaller bins in the vertical direction and the density is assumed to be uniform in that small strip, and the pair distribution function is calculated for each of these bins. However, since the number of the larger particles is small, the pair distribution function for the larger particles was determined at the location of the maximum in the density profile, and the pair distribution function was determined only as a function of horizontal separation. Figure 9 shows the pair 


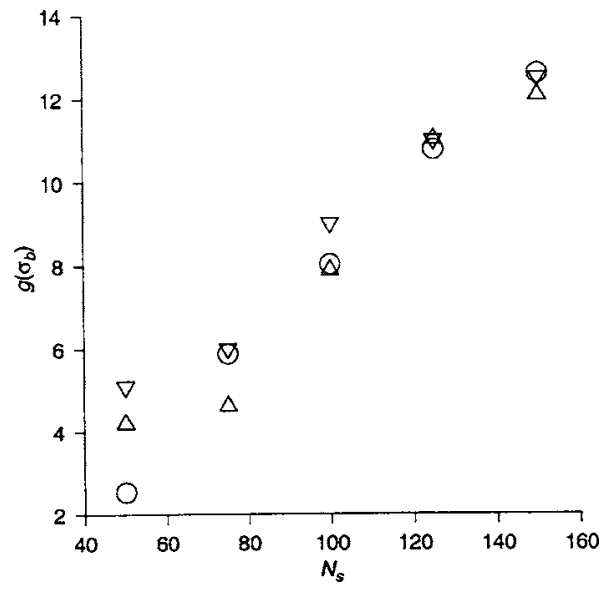

FIG. 10. Pair distribution function at contact for larger particles obtained from the model $(\bigcirc)$, experiment $(\triangle)$, and simulation $(\nabla)$ for different numbers of the smaller particles.

distribution function for the smaller particles at two different locations, and the pair distribution function for the larger particles when the number of smaller particles is 75 . The points show the results from experiments, and the lines show the predictions of the Verlet-Levesque pair correlation function for a two-dimensional hard sphere fluid at equal area fraction. The scatter in the plots provides an indication that the error bar in the experimental result is about $15 \%$ of the value of the pair distribution function, but despite this, there is very good agreement between Verlet-Levesque pair correlation function and experiments. The pair distribution function for the smaller particles is not very different from the value expected for a gas of hard spheres at the local density, indicating that the presence of the large particles does not significantly affect the interactions between the small particles. However, the pair distribution function at contact for the larger particles is significantly larger than that expected for a gas of hard spheres of large particles in the absence of small particles, indicating that the presence of smaller particles induces an attractive force between the large particles. Figure 10 shows $g_{\mathrm{bb}}\left(\sigma_{b}\right)$ obtained from the simulation as well as from the experiment, for different values of the number density of the smaller particles. It is observed that the pair distribution function at contact increases as the number of small particles is increased.

The large value of the pair distribution function at contact is due to the excluded volume effect caused by the presence of the smaller particles in the mixture and an attractive potential for a solution of macromolecules with suspended particles has been predicted [20] due to the excluded volume interaction. When two of the large particles approach each other, their excluded volumes overlap, and the effective volume available for the smaller particle increases causing an increase in the entropy and a decrease in the free energy. This leads to an effective attractive force between the particles.

The system of present interest is not a thermodynamic one, and we attempt to derive the attractive potential for a two-dimensional hard disk mixture from force balance considerations. There is an excluded area around the big particle

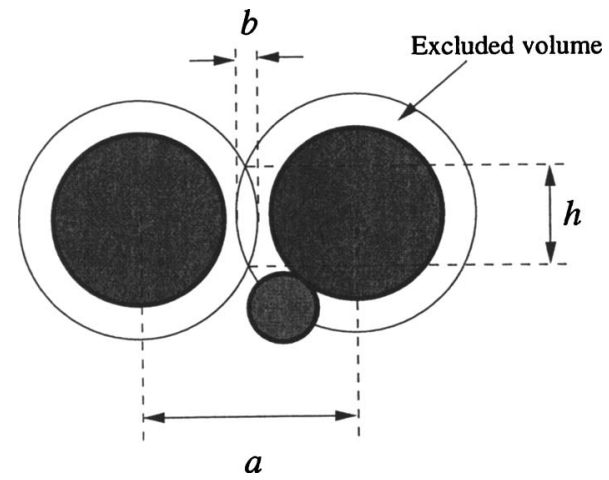

FIG. 11. Excluded volume and its overlap. The annular area around the big particle is the volume excluded that is excluded to the small particle and the area enclosed by the rectilinear lengths $b$ and $h$ is the overlap area, where $b=\sigma_{\mathrm{b}}+\sigma_{\mathrm{s}}-a$ and $h$ $=\sqrt{\left(\sigma_{\mathrm{b}}+\sigma_{\mathrm{s}}\right)^{2}+a^{2}}$, and $\sigma_{\mathrm{b}}$ and $\sigma_{\mathrm{s}}$ are the diameters of the large and the small particles, respectively.

shown in Fig. 11 where the small particles cannot enter. When two big particles approach, the excluded areas for two particles overlap, as shown in Fig. 11. There is a difference in force between the inner and outer half of the big particle, given by $p h$, where $p$ is the pressure exerted by the collisions of the small particles with the large particle. The potential is derived from the force by integrating over distance,

$$
U(a)=\int_{\sigma_{\mathrm{b}}+\sigma_{\mathrm{s}}}^{a} d x h(x) p
$$

where $p=n_{s} T$ in the dilute limit, $n_{s}$ is the number of smaller particles per unit area, and $h(x)$ is the height of the excluded volume region when the distance between centers is $x$, as shown in Fig. 11. Note that $U_{a}$ is always negative, which implies that the force is attractive. When the area fraction of smaller particles is high, the pressure is given by the equation of state

$$
p=n_{s} T\left[1+2 \nu_{s} g_{s s}\left(\nu_{s}\right)\right],
$$

where $\nu_{s}$ the are fraction of particles, and $g_{s s}(\nu)$ is the pair distribution function for the small particles at the area fraction $\nu_{s}$, and is assumed to be of the Verlet-Levesque form [Eq. (2)]. The temperature and area fraction in Eq. (6) are obtained from the simulations. When the pressure is varying with height, the potential energy is given by

$$
U(a)=\int_{\sigma_{\mathrm{b}}+\sigma_{\mathrm{s}}}^{a} d x \int_{0}^{h(x)} d z p(z) .
$$

The pair distribution function at contact (at $a=\sigma_{\mathrm{b}}$ ) for the big particles can now be evaluated. Since the density of the large particles at the center of mass location is between about 0.1 and 0.2 (Fig. 5), the pair distribution function is calculated using Eq. (4), and the potential is calculated using Eq. (7). The comparison between the simple model based on the excluded volume attraction, experiment, and simulation are shown in Fig. 10. It is observed that there is good agreement between the model predictions and simulations at all values of $N_{s}$ except for $N_{s}=50$. However, for $N_{s}=50$, it was ob- 
served that the location of the large particles is near the vibrating base, and this may be the cause of some disagreement. In all other cases, it is observed the there is good agreement between model prediction and experiment with no adjustable parameters, though the density and temperature profiles obtained from simulations have been used in calculating the pair potential. This indicates that the model accurately captures the reason for the increase in the pair distribution function.

\section{CONCLUSION}

The behavior of granular mixtures subject to vertical vibration has been studied. Two interesting features have been observed, the rise of the larger particles in a sea of smaller particles and an apparent increase in the "attraction potential" between two large particles. The rise of the larger par- ticle in a background of the smaller particles is due to the difference in the force exerted by smaller particles on the top and the bottom halves of the larger particles. The mean position of the larger particles is determined from the force balance on the larger particles. The position obtained from the simulation is in good agreement with that predicted from the force balance treatment in most of the cases. However, in the cases where the average height of the larger particle is near the vibrating base, the agreement is poor. The increase in the pair distribution function is attributed to the excluded volume effect on the larger particles due to the presence of the smaller particles and an expression for the pair distribution function is derived from the excluded volume consideration. The model predictions are in good agreement with the observations in experiments and simulation with no adjustable parameters when the number of small particles is high, indicating that the simple model accurately captures the physical mechanism for the attractive force.
[1] A. Rosato, K. J. Strandburg, F. Prinz, and R. H. Swendsen, Phys. Rev. Lett. 58, 1038 (1987).

[2] W. Cooke, S. Warr, J. M. Huntley, and R. C. Ball, Phys. Rev. E 53, 2812 (1996).

[3] J. Duran, J. Rajchenbach, and L. Clement, Phys. Rev. Lett. 70, 2431 (1993).

[4] R. Jullien, P. Meaking, and A. Pavlovitch, Phys. Rev. Lett. 69, 640 (1992).

[5] J. B. Knight, H. M. Jaeger, and S. R. Nagel, Phys. Rev. Lett. 70, 3728 (1993).

[6] M. E. Mobius, L. E. Benjamin, S. R. Nagel, and H. M. Jaeger, Nature (London) 414, 270 (2001).

[7] T. Shinbrot and F. J. Muzzio, Phys. Rev. Lett. 81, 4365 (1998).

[8] D. C. Hong and P. V. Quinn, Phys. Rev. Lett. 86, 3423 (2001).

[9] A. P. J. Breu, H. M. Ensner, C. A. Kruelle, and L. Rehberg, Phys. Rev. Lett. 90, 014302 (2003).

[10] K. Feitosa and N. Menon, Phys. Rev. Lett. 88, 198301 (2002).

[11] R. D. Wildman and D. J. Parker, Phys. Rev. Lett. 88, 064301 (2002).

[12] M. Alam, J. T. Willits, B. O. Aranson, and S. Luding, Phys.
Fluids 14, 4085 (2002).

[13] S. R. Dahl, C. M. Hrenya, G. Garzo, and J. W. Dufty, Phys. Rev. E 66, 051303 (2002).

[14] A. Barrat and E. Trizac, Phys. Rev. E 66, 051303 (2002).

[15] T. Mullin, Science 295, 1891 (2002).

[16] P. M. Reis and T. Mullin, Phys. Rev. Lett. 89, 244301 (2002).

[17] S. Aumaitre, C. A. Kruelle, and I. Rehberg, Phys. Rev. E 64, 041305 (2001).

[18] R. Pagnani, U. M. B. Marconi, and A. Puglisi, Phys. Rev. E 66, 051304 (2002).

[19] N. Sisodia and C. Wassgren, Phys. Rev. Lett. 87, 084302 (2001).

[20] S. Asakura and F. Oosawa, J. Polym. Sci. XXXIII, 183 (1958).

[21] P. Sunthar and V. Kumaran, Phys. Rev. E 64, 041303 (2001).

[22] M. P. Allen and D. J. Tildesley, Computer Simulation of Liquids (Clarendon Press, Oxford, 1990).

[23] J.-P. Hansen and I. McDonald, Theory of Simple Liquids (Academic Press, London, 1990).

[24] L. Verlet and D. Levesque, Mol. Phys. 46, 969 (1982). 